\title{
Gene Exchange Operators of Partheno-Genetic Algorithm for Permutation Flowshop Scheduling with Maximum and Minimum Time Lag Constraints
}

\author{
Bailin Wang ${ }^{1,2, a}$, Haifeng Wang ${ }^{1,2, b}$, Tieke $\mathrm{Li}^{1,2, \mathrm{c}}$ \\ ${ }^{1}$ Donlinks School of Economics and Management, University of Science and Technology Beijing, \\ Beijing, 100083, China \\ ${ }^{2}$ Engineering Research Center of MES Technology for Iron \& Steel Production, Ministry of \\ Education, Beijing, 100083, China \\ aemail: wangbl@ustb.edu.cn, ${ }^{\text {bemail: wanghf@ustb.edu.cn, }{ }^{c} e m a i l: ~ t i e k e @ u s t b . e d u . c n, ~}$
}

\begin{abstract}
Keywords: Permutation Flowshop Scheduling; Time Lags; Partheno-Genetic Algorithm; Gene Exchange Operator
\end{abstract}

\begin{abstract}
Permutation flowshop scheduling problem with maximum and minimum time lag constraints to minimize the makespan is considered, and a framework of partheno-genetic algorithm for the problem is presented. Based on the algorithm framework, two gene exchange operators are discussed, including single-point exchange operator and multi-point exchange operator. Experimental results show that if the single-point exchange operator is adopted in the parthenogenetic algorithm, the performance is good in both effectiveness and efficiency.
\end{abstract}

\section{Introduction}

In some industrial manufacturing environments with high production continuity, there are typical constraints named maximum and minimum time lag constraints: Due to the instable physical or chemical nature of intermediate products, there exist maximum time lag constraints that the waiting time (time lag) of each job between two consecutive production processes must not be larger than a given upper bound. Moreover, because of the transportation requirements of jobs from one process to the next process, minimum time lag constraints which restrict minimum values of waiting times are considered. Flowshop is one of the most fundamental multi-process production environments with strong industrial background, and the study on the flowshop scheduling problem with these time lag constraints could provide theoretical basis for practical production.

The permutation scheduling problem with maximum and minimum time lag constraints was first discussed by Fondrevelle, Oulamara and Portman in 2006 [1]. They proved that even the problem with two machines to minimize the makespan is strongly NP-hard, and presented a brand and bound method. In 2008 [2], the three scholars further considered the objective of minimum weighted sum of machine completion times, and proposed a branch and bound procedure. In 2009 [3], they investigated the problem with exact time lags to minimize the maximum lateness, and a branch and bound method was designed. In addition, Dhouib, Teghem and Loukil [4] studied the problem with the objective to minimize the tardy job number and the makespan. The problem model was presented as a mixed integer programming, and several versions of simulated annealing algorithm were proposed. Hamdi and Loukil discussed the problem with exact time lags to minimize the total earliness and tardiness, and gave two upper bounds and two lower bounds of the optimal value by scheduling rules [5].

At present, the study on the permutation scheduling problem with maximum and minimum time lags is still in an initial stage. In this paper, a permutation flowshop scheduling problem with maximum and minimum time lags to minimize the makespan is considered. An algorithm framework of partheno-genetic algorithm is presented and two gene exchange operators are proposed. Finally, an evaluation of the performances of gene exchange operators is carried out by computational experiments. 


\section{Problem Description}

The permutation flowshop scheduling problem with maximum and minimum time lags considered here can be descript as follows. There are $n$ jobs $\left\{J_{1}, J_{2}, \ldots, J_{n}\right\}$ which have to be processed on $m$ machines $\left\{M_{1}, M_{2}, \ldots, M_{m}\right\}$ via the same route $\left(M_{1} \rightarrow M_{2} \rightarrow \ldots \rightarrow M_{m}\right)$. The same processing sequence of jobs is maintained throughout. Upper bounds and lower bounds of waiting times of jobs between consecutive processes are restricted. The problem is to find a processing sequence of jobs and determine starting and completion times, such that the makespan, which is equal to the maximum completion time, is minimized.

The following notations are used throughout the paper.

$\boldsymbol{J}_{\boldsymbol{i}}$ : job $i$, where $i$ is the job number, $i=1, \ldots, n$. $\boldsymbol{M}_{j}$ : the $j$-th machine, where $j$ is the machine number, $j=1, \ldots, m$. $\boldsymbol{O}_{i j}$ : the operation of $J_{i}$ on $M_{j}$, where $i=1, \ldots, n ; j=1, \ldots, m$.

$\boldsymbol{p}_{i j}$ : processing time of $O_{i j}$, where $i=1, \ldots, n, j=1, \ldots, m . \boldsymbol{w}_{i j}, \boldsymbol{\alpha}_{i j}, \boldsymbol{\beta}_{i j}$ : waiting time and its maximum and minimum bounds of $J_{i}$ between $M_{j}$ and $M_{j+1}$ respectively, where $i=1, \ldots, n ; j=1, \ldots, m-1$.

$\boldsymbol{\pi}$ : job sequence to be processed; $\boldsymbol{\pi ( i )}$ : the number of $i$-th job in $\pi$, where $i=1, \ldots, n$.

$S_{i j}, C_{i j}$ : starting time and completion time of $O_{i j}$ respectively, where $i=1, \ldots, n ; j=1, \ldots, m$.

$C_{\max }$ : the makespan, where $C_{\max }=\max \left\{C_{i j}\right\}$, and the optimization objective is $\min C_{\max }$.

Based on the above notations, the problem can be denoted by Fm $\mid$ prmu, $\beta_{i j} \leq w_{i j} \leq \alpha_{i j} \mid C_{\max }$.

\section{Framework of a Partheno-Genetic Algorithm}

Partheno-Genetic Algorithm (PGA) is proposed as an improved Genetic Algorithm (GA) by Li and Tong in 1999 [6]. In PGA, crossover operator is no longer used. Instead, gene recombination operator and gene mutation operator are proposed as parthenogenesis to generate offspring. At present, PGA has been applied to many areas [7], production planning [8] and production scheduling [9].

For the considered scheduling problem, the following aspects are focused on for PGA design: encoding mode and population size, initial population generation approach, genetic operators, selection operator, and algorithm termination conditions. Concrete solutions for these aspects are given below.

1)Encoding mode and Population Size: Partheno-genetic algorithm has a strong advantage to deal with problems with the solution representation as series number string. Hence, for the considered problem, job numbers are taken as gene codes to form a chromosome. Each chromosome represents a feasible job sequence with the length $n$. The population size is equal to the length of a chromosome $n$.

2)Initial population generation approach: The well-known heuristic NEH is applied to generate initial population in this algorithm, and the process to calculate the makespans of partial schedules are improved with the consideration of maximum and minimum time lag constraints.

3)Selection operator: Tournament method is applied in this algorithm as the selection operator to choose the top $n$ individuals for the optimization objective makespan from all the parents and their offsprings to form a new population.

4)Algorithm termination condition: The termination condition of the PGA proposed in this paper is set as: If the current number of iterations is larger than $5 \times n$ then the algorithm is terminated.

5)Genetic operator design: The main genetic operators in a parthenon genetic algorithm may be divided into two types, gene recombination operator and gene mutation operator. For the considered problem, because the codes of genes in a chromosome are all different, only gene recombinant operators can be adopted.

There are mainly three kinds of gene recombination operators: gene exchange operators, gene shift operators and gene inverse operators. The construction strategies of gene exchange operators will be focused on in the rest of this paper. 


\section{Gene Exchange Operators}

Gene exchange operator is the process to exchange genes in one or more pairs of positions in a chromosome. Two gene exchange operators are discussed here.

\section{1)Single-point exchange operator}

Single-point exchange operator is to select a pair of positions randomly in the chromosome and exchange genes in the two positions each time.

For example, given an individual with the job sequence $\{3,1,4,5,2\}$, if genes in the positions 1 and 4 are chosen to be exchanged by single-point exchange operator, the new chromosome is $\{5,1$, $4,3,2\}$.

\section{2)Multi-point exchange operator}

Multi-point exchange operator is to select multiple pairs of positions randomly in the chromosome to exchange these genes each time.

Taken the above example as well, a two-point exchange operator is carried out for the individual $\{3,1,4,5,2\}$, and two pairs of positions are selected as (1,3)(2,5). After exchanging genes in these positions, a new chromosome is generated as $\{4,2,3,5,1\}$.

For the considered problem, the rules for multi-point exchange operator are defined as follows.

Rule 1: The number of exchange pairs are generated randomly with the minimum value 1 and the maximum value $\lfloor n / 2\rfloor$.

Rule 2: In the union of all the pairs, the same positions are not allowed.

Corresponding to the above example, because $n=5$, according to Rule 1 , the multi-point exchange operator carried out each time may be a single-point or two-point exchange operator; according to Rule 2, the two or four exchanging positions must be all different to ensure the exchange operation feasible.

\section{Computational Experiments}

In this section, computational experiments are carried out to compare the optimization performances of the two gene exchange operators. For clarity, algorithms with the three operators are respectively named as SPGA (short for Single-Point Exchange Operator based PGA) and MPGA (short for Multi-Point Exchange Operator based PGA). The two algorithms are coded in C\# language and implemented on the computer with Intel Core i3-4020Y/ CPU1.5GHz/ RAM4.0G. The parameters of test problems are set as $n=\{20,40,60,80,100\}, m=\{2,4,6,8\}, p_{i j}, \alpha_{i j}$ and $\beta_{i j}$ are all belong to $D U[1,99]$, and $\beta_{i j} \leq \alpha_{i j}$. According to the different problem sizes, the experiments are divided into 20 groups and each group generated 10 test problems randomly, thus there are a total of 200 instances. The following two kinds of performance measurements are considered here.

The first one is the improving ratio on the makespan of the initial solution obtained by $\mathrm{NEH}$, denoted by $R$. Its computing formula is shown below, where $C_{\max }^{*}$ is the best makespan obtained by the corresponding PGA algorithm, and $C_{\max }^{0}$ is the makespan of the initial solution.

$$
R=\frac{C_{\max }^{0}-C_{\max }^{*}}{C_{\max }^{0}} \times 100 \%
$$

The other measurement is the computing time, denoted by $T$.

Computational results are shown in Table 1. These data demonstrate following points.

Concerning the computational efficiency, computing times of algorithms with the two operators had no significant differences. Computing times of SPGA were little faster than the other algorithm MPGA.

Concerning the optimization effect, single-point exchange operator was evidently superior to multi-point exchange operator. Moreover, in the 200 instances generated randomly, the best improving ratios of SPGA and MPGA were $6.48 \%$ and $3.32 \%$ respectively. It indicates that single-point exchange operator has better adaptability for the considered problem. 
Table 1 Computing results

\begin{tabular}{|l|l|l|l|l|l|l|l|l|c|}
\hline \multirow{2}{*}{$n \times m$} & \multicolumn{2}{|c|}{ SPGA } & \multicolumn{2}{c|}{ MPGA } & \multirow{2}{*}{$n \times m$} & \multicolumn{2}{c|}{ SPGA } & \multicolumn{2}{c|}{ MPGA } \\
\cline { 2 - 6 } \cline { 8 - 10 } & $\bar{R} / \%$ & $\bar{T} / \mathrm{s}$ & $\bar{R} / \%$ & $\bar{T} / \mathrm{s}$ & & $\bar{R} / \%$ & $\bar{T} / \mathrm{s}$ & $\bar{R} / \%$ & $\bar{T} / \mathrm{s}$ \\
\hline $20 \times 2$ & 0.45 & 0.02 & 0.37 & 0.03 & $60 \times 6$ & 1.20 & 1.56 & 0.16 & 1.81 \\
\hline $20 \times 4$ & 1.79 & 0.04 & 0.75 & 0.05 & $60 \times 8$ & 0.77 & 2.05 & 0.22 & 2.21 \\
\hline $20 \times 6$ & 1.46 & 0.06 & 0.41 & 0.06 & $80 \times 2$ & 0.12 & 1.08 & 0.03 & 1.64 \\
\hline $20 \times 8$ & 1.39 & 0.08 & 0.57 & 0.09 & $80 \times 4$ & 0.85 & 2.26 & 0.21 & 2.79 \\
\hline $40 \times 2$ & 0.37 & 0.16 & 0.14 & 0.23 & $80 \times 6$ & 0.93 & 3.32 & 0.27 & 3.78 \\
\hline $40 \times 4$ & 1.51 & 0.30 & 0.40 & 0.36 & $80 \times 8$ & 0.80 & 4.44 & 0.22 & 4.95 \\
\hline $40 \times 6$ & 1.52 & 0.57 & 0.52 & 0.62 & $100 \times 2$ & 0.33 & 1.87 & 0.14 & 2.75 \\
\hline $40 \times 8$ & 1.52 & 0.60 & 0.44 & 0.67 & $100 \times 4$ & 0.91 & 4.02 & 0.12 & 4.93 \\
\hline $60 \times 2$ & 0.15 & 0.45 & 0.09 & 0.65 & $100 \times 6$ & 0.59 & 6.13 & 0.14 & 7.09 \\
\hline $60 \times 4$ & 1.12 & 0.99 & 0.22 & 1.22 & $100 \times 8$ & 0.62 & 8.37 & 0.11 & 9.30 \\
\hline
\end{tabular}

To sum up, for the considered scheduling problem, the presented framework of partheno-genetic algorithm is feasible, and it would perform well in both effectiveness and efficiency while the single-point exchange operator is applied.

\section{Conclusion}

Permutation flowshop scheduling problem with maximum and minimum time lag constraints is a common production management problem in process industry with requirements of transportation and continuous production. For solving the problem effectively, a framework of partheno-genetic algorithm is proposed, and two gene exchange operators are put forward as single-point exchange operator and multi-point exchange operator. Experimental results show that the presented framework of partheno-genetic algorithm is feasible, and it will have good performance in both effectiveness and efficiency if the single-point exchange operator is used. This work may provide the basis for further design of more optimized partheno-genetic algorithm for the problem.

\section{Acknowledgement}

This work was financially supported by China Scholarship Council, Fundamental Research Funds for the Central Universities (No. FRF-BR-14-011A), and China Postdoctoral Science Foundation (No. 2012M510324).

\section{References}

[1] J. Fondrevelle, A. Oulamara, M. C. Portmann. Permutation flowshop scheduling problems with maximal and minimal time lags. Computers \& Operations Research, 2006, 33: 1540-1556.

[2] J. Fondrevelle, A. Oulamara, M. C. Portmann. Permutation flowshop scheduling problems with time lags to minimize the weighted sum of machine completion times. International Journal of Production Economics, 2008, 112: 168-176.

[3] J. Fondrevelle, A. Oulamara, M. C. Portmann, A. Allahverdi. Permutation flowshops with exact time lags to minimize maximum lateness. International Journal of Production Research, 2009, 47: 6759-6775.

[4] E. Dhouib, J. Teghem, T. Loukil. Lexicographic optimization of a permutation flow shop scheduling problem with time lag constraints. International Transactions in Operational Research, 2013, 20: 213-232. 
[5] I. Hamdi, T. Loukil. Bounds for the permutation flowshop scheduling problem with exact time lags to minimize the total earliness and tardiness. International Journal of Combinatorial Optimization Problems and Informatics, 2014, 5: 47-57.

[6] M. J. Li, T. S. Tong. A partheno genetic algorithm and analysis on its global convergence. Acta Automat Sin, 1999, 25: 68-72.

[7] J. Wang, W. Huang, G. Ma, S. Chen. An improved partheno genetic algorithm for multiobjective economic dispatch in cascaded hydropower systems. International Journal of Electrical Power \& Energy Systems, 2015, 67: 591-597.

[8] C. Wang, Q. Liu, Q.-Y. Li, et al. Optimal charge plan model for steelmaking based on modified partheno-genetic algorithm. Control Theory and Applications, 2013, 30: 734-741.

[9] L. Wang, D.-B. Tang. An improved adaptive genetic algorithm based on hormone modulation mechanism for job-shop scheduling problem. Expert Systems with Applications, 2011, 38: 7243-7250. 